Classification

Physics Abstracts

$31.30 \mathrm{~N}-63.20 \mathrm{~K}-75.10 \mathrm{H}-33.40$

\title{
A Simple Ising-Like Model for Spin Conversion Including Molecular Vibrations
}

\author{
A. Bousseksou $\left({ }^{1}\right), H$. Constant-Machado $\left({ }^{2, *}\right)$, F. Varret $\left({ }^{3}\right)$ \\ ( ${ }^{1}$ Laboratoire de Chime de Coordination du C.N.R.S., 205 Route de Narbonne, 31077 Toulouse \\ Cédex, France \\ $\left({ }^{2}\right)$ Département de Recherches Physiques $\left({ }^{* *}\right)$, Université Pierre et Marie Curıe, 75252 Paris \\ Cedex 05, France \\ $\left({ }^{3}\right)$ Laboratoire de Magnétisme et d'Optique $\left({ }^{* * *}\right)$, Université de Versailles Saint-Quentin, 78035 \\ Versailles Cédex, France
}

(Recelved 6 June 1994, revised and accepted 9 January 1995)

\begin{abstract}
An Ising-type model for spin conversion, explicitly accounting for intramolecular vibrations has been studied. Each two level system is associated with $p$ harmonic oscllators having two possible frequencies $\omega_{\mathrm{LS}}^{(i)}, \omega_{\mathrm{HS}}^{(i)}$. The major advantage of this model is that it provides an excellent agreement with both the conversion curve and calorimetric data, in particular the entropy change upon spin conversion.

With the help of Arrhenius plots, the predictions of the model are accurately compared to the literature experimental data on the spin equilibrium curves. A very accurate Mössbauer investigation by Jacobi, Spierıng and Gütlich, provıdes evidence for a small effect typical for vibrations. A novel example is given where the conversion curve is essentially monitored by vibrations; this originates from the extremely small value of the energy gap between HS and LS electrovibrational groundstates.

However, in most cases, as a first approach, the low frequency approximation of the model can be used. Then the model reduces to a simple two-level model with additional degeneracies implicitely accounting for intramolecular vibrations.
\end{abstract}

\section{Introduction}

Among the theoretical models developed to describe the thermodynamics of conversion between high spin (HS) and low spin (LS) states, the two-state models [1] or two-level models [2-4] are widely used because of their practical convenience.

$\left({ }^{*}\right)$ on leave from Universidad Central of Venezuela

$\left({ }^{* *}\right)$ CNRS URA $n^{\circ} 71$

$\left({ }^{* * *}\right)$ CNRS URA n ${ }^{\circ} 1531$

(C) Les Editions de Physique 1995 
The two-state macroscopic description [1] considers two "spin isomers" in chemical equilibrium, according to the reversible reaction:

$$
\mathrm{LS} \rightleftharpoons \mathrm{HS} \quad\left(\Delta S^{0}, \Delta H^{0}\right)
$$

In absence of cooperativity, $\Delta S^{0}, \Delta H^{0}$ do no depend on the molar fractions $n_{\mathrm{HS}}, n_{\mathrm{LS}}$.

The importance of molecular vibrations in the spin conversion problem was first recognized by Sorai and Seki [5] on the basis of calorimetric measurements. Indeed, the purely electronic models [1-4] resulted in a value of the molar entropy change upon conversion, $\Delta S_{\text {electr }} \simeq \Delta S_{\text {spin only }}=13.4 \mathrm{~J} \mathrm{~K}^{-1} \mathrm{~mol}^{-1}$, far below all experimental data, ranging from 35 to $80 \mathrm{~J} \mathrm{~K}^{-1} \mathrm{~mol}^{-1}$. The missing entropy was attributed to vibrational effects, mostly of intramolecular origin. Further studies provided evidence for spectacular changes in the vibrational spectra, together with a minor change in the Debye temperature, observed by Mössbauer spectroscopy [6]. Recent reviews of all the corresponding data are given in the references $[7,8]$.

The first model explicitly accounting for vibrations was introduced by Zimmermann and König [9]. Its main goal was to quantitatively account for the molar entropy increase upon spin conversion, $\Delta S$. The model includes ligand field and spin-orbit coupling, but these ingredients did not yield any specific effect. The vibration modes are assumed to follow the Debye model, 1rrespectively of their intra- or intermolecular character; the Debye temperature is assumed to vary as $\sqrt{\Theta_{\mathrm{LS}}^{2}+\left(\Theta_{\mathrm{HS}}^{2}-\Theta_{\mathrm{LS}}^{2}\right) n_{\mathrm{HS}}}$. This results in a contribution to the free energy $\sim$ $n_{\mathrm{HS}}^{2}$, thus equivalent to an intermolecular coupling through the phonons. The total molar entropy variation calculated using $\Theta_{\mathrm{LS}}=140 \mathrm{~K}, \Theta_{\mathrm{HS}}=130 \mathrm{~K}$, reaches the correct magnitude (36 J.K $\mathrm{K}^{-1} \mathrm{~mol}^{-1}$ ). In the same paper the authors have shown the formal equivalence between two-level models treated in the mean-field approximation, and macroscopic two states models based on the thermodynamics of regular solutions in the Bragg-Williams approximation.

Another model explicitly including vibrations has been developed by Kambara [10]; this vibronic model is based on the coupling of the electronic state with both intramolecular vibrations and a lattice strain. Kambara's model also yields qualitatively correct results; however, to our knowledge, it has not been used to fit experimental data, probably because of the detailed nature of the needed calculations.

Recently, we proposed [4] taking advantage of the formal relation:

$$
\Delta S^{0}=R \operatorname{Ln} \frac{g_{\mathrm{HS}}}{g_{\mathrm{LS}}}
$$

where $g_{\mathrm{HS}}$ et $g_{\mathrm{LS}}$ are the degeneracies associated with the two spin states. "Effective" values of the degeneracy ratio much higher than the pure spin value $(2 s+1)$, are needed to reach the experimental $\Delta S$ values. This increase in degeneracies merely accounts for the density of vibrational states much higher in the HS than in the LS state. Values of the ratio as high as $\simeq 500-2000$ led to good fits [11] of the experimental $n_{\mathrm{HS}}(T)$ curves, much improved for their high-temperature branches, while the experimental values of the molar entropy were correctly obtained. The approximations behind such a procedure will be analyzed here.

Here we shall deal only with the intramolecular vibrations, since the intermolecular vibration contribution to $\Delta S$ has been estimated $[5,6]$ to be of minor importance, compared to the intramolecular contribution. The vibrational effects will be better characterized in absence of cooperativity.

This reported is organized as follows:

Section 2: brief review of the calorimetric aspects;

Section 3: short description of the "Ising-like" model;

Section 4: the model including vibrations; 


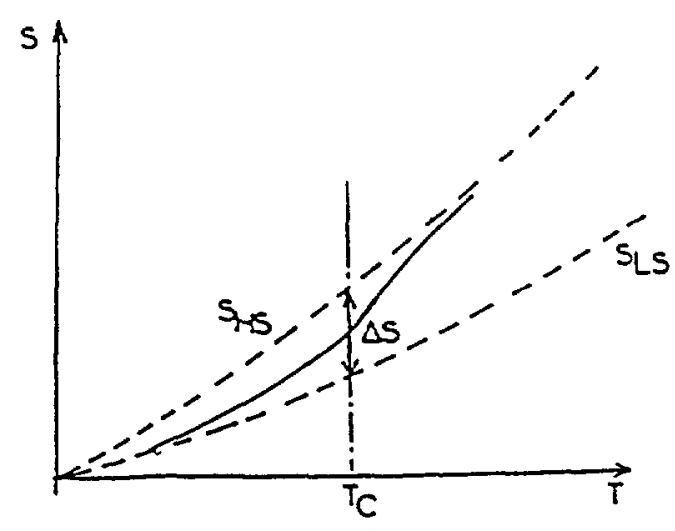

a)

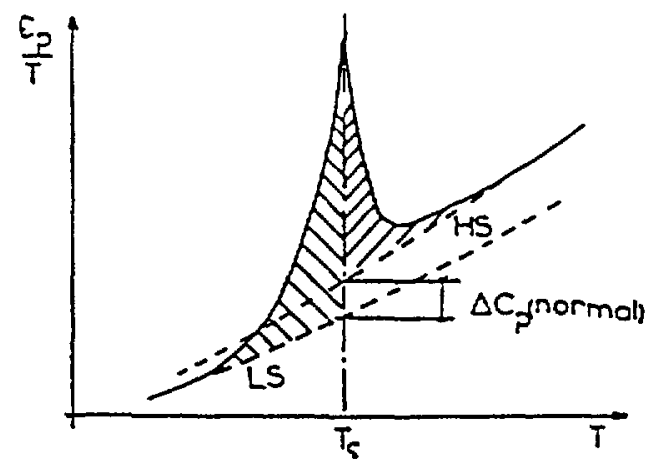

b)

Fig. 1. - a) thermal variation of the molar entropy in the case of spin conversion, compared to the variations associated with pure LS and HS states. b) corresponding variation of the specific heat, plotted as $\frac{C_{P}}{T}$ vs. $T$.

Section 5: results;

Section 6: discussion and analysis of experimental data.

\section{Calorimetric Aspect}

It is useful to precisely define the molar entropy increase upon conversion $\Delta S(T)$. Considering the spin conversion as a reversible chemical reaction, then (per mole):

$$
\Delta S(T)=S_{\mathrm{HS}}(T)-S_{\mathrm{LS}}(T)
$$

where $S_{\mathrm{HS}}(T), S_{\mathrm{LS}}(T)$ are the molar entropies the compound would have at temperature $T$ if it were entirely in the high-spin or low-spin state, respectively. Very likely, the molar entropy of the compound, $S(T)$, is between $S_{\mathrm{LS}}(T)$ and $S_{\mathrm{HS}}(T)$; in any case, when a complete spin conversion occurs, it varies from the low-spin value at low temperature to the high-spin value at high temperature. This is schematized in Figure 1a. The corresponding specific heat curve is shown in Figure $1 \mathrm{~b}$, given as $\frac{C_{\mathrm{P}}}{T}=\frac{\mathrm{d} S}{\mathrm{~d} T}$ vs. $T$.

The equilibrium temperature $T_{\mathrm{C}}$ defined by $n_{\mathrm{HS}}=n_{\mathrm{LS}}=\frac{1}{2}$, is such that $\Delta G\left(T_{\mathrm{C}}\right)=0$; consequently:

$$
\Delta H\left(T_{\mathrm{C}}\right)=T_{\mathrm{C}} \Delta S\left(T_{\mathrm{C}}\right)
$$

The "normal" regimes (i.e., those of the pure LS or HS states), can be extrapolated to the equilibrium temperature from each side; this leads to the discontinuity $\Delta C_{\mathrm{P}}^{\text {normal }}$ evidenced by Sorai and Seki [5] in the case of a true spin transition, characterized by a discontinuity of $n_{\mathrm{HS}}(T)$. The discontinuity in the normal specific heat provided the first experimental evidence for the electron-vibrational nature of the spin conversion.

When the spin conversion is complete, $\Delta S\left(T_{\mathrm{C}}\right)$ is easily deduced from the plot of $\frac{C_{\mathrm{P}}}{T}$ vs. $T$. This is demonstrated in the following way: 


$$
\begin{gathered}
\Delta S\left(T_{\mathrm{c}}\right)=S_{\mathrm{HS}}\left(T_{\mathrm{c}}\right)-S_{\mathrm{LS}}\left(T_{\mathrm{c}}\right) \\
=S\left(T_{\mathrm{c}}\right)-S_{\mathrm{LS}}\left(T_{\mathrm{c}}\right)+S_{\mathrm{HS}}\left(T_{\mathrm{c}}\right)-S\left(T_{\mathrm{c}}\right)
\end{gathered}
$$

Assuming $S\left(T^{\prime}\right)=S_{\mathrm{HS}}\left(T^{\prime}\right)$ (complete conversion for $T^{\prime} \gg T_{\mathrm{c}}$ )

then:

$$
S(0)=S_{\mathrm{LS}}(0) \text { (third thermodynamical law) }
$$

$$
\Delta S\left(T_{\mathrm{C}}\right)=\int_{0}^{T_{\mathrm{C}}}\left(\mathrm{d} S(T)-\mathrm{d} S_{\mathrm{LS}}(T)\right)+\int_{T_{\mathrm{C}}}^{T^{\prime}}\left(\mathrm{d} S(T)-\mathrm{d} S_{\mathrm{HS}}(T)\right)
$$

Relation (5) means that $\Delta S\left(T_{\mathrm{c}}\right)$ is obtained by summing up the hatched areas of Figure 1 (b).

\section{The Two-Level (Ising-Like) Model}

The two-level hamiltonian, including cooperativity in mean-field approach, is written

$$
\hat{\mathcal{H}}=\frac{1}{2} \Delta_{0} \hat{\sigma}-J \hat{\sigma}<\hat{\sigma}>
$$

where $\Delta_{0}$ is the energy spacing of the isolated two-level system and $\hat{\sigma}$ a fictitious spin operator taking the values $-1,+1$ for the spin state LS and HS, respectively; then the high-spin fraction is derived as: $n_{\mathrm{HS}}=\frac{\langle\hat{\sigma}\rangle+1}{2}$

Resolving $\mathcal{H}$ in canonical statistics yields:

$$
\frac{n_{\mathrm{HS}}}{1-n_{\mathrm{HS}}}=K_{\text {eq }}=\frac{g_{\mathrm{HS}}}{g_{\mathrm{LS}}} \mathrm{e}^{-\frac{\Delta\left(n_{\mathrm{HS}}\right)}{k_{\mathrm{BT}}}}
$$

with

$$
\left.\Delta\left(n_{\mathrm{HS}}\right)=\Delta_{0}-2 J<\hat{\sigma}\right\rangle=\Delta_{0}+2 J-4 J n_{\mathrm{HS}}
$$

Equation (7) is formally equivalent to the mass action law:

$$
K_{\text {eq }}=\mathrm{e}^{-\frac{\Delta H}{R T}+\frac{\Delta S_{0}}{R}}
$$

written here assuming the interaction to be purely enthalpic.

By identifying equations (7) and (9), an interesting expression is obtained for the molar entropy change upon (complete) conversion:

$$
\Delta S^{0}=R \operatorname{Ln} \frac{g_{\mathrm{HS}}}{g_{\mathrm{LS}}}
$$

A second useful relation is easily derived from equations (7) and (8), dealing with the equilibrium temperature $\left(n_{\mathrm{HS}}=1 / 2\right)$, holding for any $J$ :

$$
\Delta_{0}=k_{\mathrm{B}} T_{\mathrm{c}} \operatorname{Ln} \frac{g_{\mathrm{HS}}}{g_{\mathrm{LS}}}
$$

Conversion curves are conveniently visualized in term of Arrhentus plots, Ln $K_{\text {eq }}=f\left(\frac{1}{T}\right)$, which are linear in the absence of interactions, and show typical S-shaped distortions when intramolecular interactions are included (see Fig. 2). 


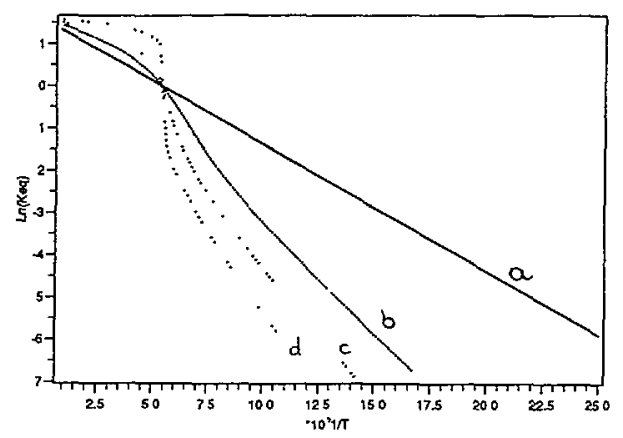

Fig. 2. - Arrhenius plots computed using equation (7), for different values of the intermolecular coupling ( here defined positive for cooperativity coupling): $J=0,110,150,220 \mathrm{~K}$ from (a) to (d). Degeneracy ratio $\frac{g_{\mathrm{HS}}}{g_{\mathrm{LS}}}=5, \Delta\left(n_{\mathrm{HS}}=\frac{1}{2}\right)=300 \mathrm{~K}$.

It will be noted that the intersect with the ordinate axis $(T \rightarrow \infty)$, directly yields, using relations (7) and (8), the value of $\Delta S^{0}$;

$$
\Delta S^{0}=R \operatorname{Ln} K_{\text {eq }}(T \rightarrow \infty)=R \operatorname{Ln} \frac{g_{\mathrm{HS}}}{g_{\mathrm{LS}}}
$$

In other words, the high temperature values of the spin populations and the molar entropy change are closely related. Indeed, experimental data associate large value of $\Delta S$ (with respect to the electronic contribution), and a quasi-complete HS character at high temperature(in excess of the electronic degeneracy ratio).

\section{Two-Level Model Including Vibrations}

Each molecule is assumed to behave as a set of $p$ independent harmonic oscillators, with frequencies $\omega_{L S}^{(2)}$ in the LS state, $\omega_{\mathrm{HS}}^{(2)}$ in the HS state.

The partition function associated with one molecule is written:

$$
\mathbb{Z}(T)=g_{\mathrm{LSE}} \mathbb{Z}_{\mathrm{vib}}^{\mathrm{LS}}(T)+g_{\mathrm{HSE}} \mathbb{Z}_{\mathrm{vib}}^{\mathrm{HS}}(T) \mathrm{e}^{-\frac{\Delta\left(n_{\mathrm{HS}}\right)}{k_{\mathrm{B}} T}}
$$

where $g_{\mathrm{LSE}}, g_{\mathrm{HSE}}$ are the electronic degeneracies of the two states. $\Delta\left(n_{\mathrm{HS}}\right)$ is the mean- field expression of the electronic contribution to the energy difference between states of different spin. Usually, $\frac{g_{\mathrm{HSE}}}{g_{\mathrm{LSE}}}=2 S+1=5$ is taken for $\mathrm{Fe}^{\mathrm{II}}$ complexes.

The vibrational partition function is written:

$$
\mathbb{Z}_{\mathrm{vib}}(T)=\prod_{\imath=1}^{p} z_{\mathrm{vib}}\left(\omega_{2}, T\right)
$$

where $z\left(\omega_{2}, T\right)$ is the partition function of the isolated harmonic $i^{\text {th }}$ oscillator (in the LS or HS state).

By thermal averaging, the equilibrium constant is obtained: 


$$
K_{\mathrm{eq}}(T)=\frac{n_{\mathrm{HS}}}{1-n_{\mathrm{HS}}}=\frac{g_{\mathrm{HSE}} \mathbb{Z}_{\mathrm{vib}}^{\mathrm{HS}}(T)}{g_{\mathrm{LSE}} \mathbb{Z}_{\mathrm{vib}}^{\mathrm{LS}}(T)} \mathrm{e}^{-\frac{\Delta(0)-4 J n_{\mathrm{HS}}}{k_{\mathrm{B}} T}}
$$

This self-consistent equation is resolved numerically (by dichotomy, to start with) and yields the thermal evolution of the system: $n_{\mathrm{HS}}(T)$ or $K_{\mathrm{eq}}(T)$. The identification of equation (15) to the mass action law is no longer as simple as in section 3 , and leads to $\Delta H$ and $\Delta S$ values which depend on temperature.

Straightforward algebraic transformations yield:

$$
K(T \rightarrow \infty)=\frac{g_{\mathrm{HSE}}}{g_{\mathrm{LSE}}} \prod_{\imath=1}^{p} \frac{\omega_{\mathrm{LS}}^{(2)}}{\omega_{\mathrm{HS}}^{(2)}}
$$

The specific heat jump and the entropy change, both for complete spin conversion, are easily calculated by difference between the pure HS and LS states.

\section{Results}

A useful particular case is developed first: the case of low frequencies.

For $\hbar \omega \ll k_{\mathrm{B}} T$ we previously noticed [4] that the ratio of the vibrational partition functions becomes independent of temperature. This comes from:

$$
z_{\mathrm{vib}}\left(\omega_{2}, T\right) \simeq \frac{k_{\mathrm{B}} T}{\hbar \omega_{2}}
$$

leading to:

$$
\left(\frac{n_{\mathrm{HS}}}{1-n_{\mathrm{LS}}}\right)_{\mathrm{LF}}=\frac{g_{\mathrm{HSE}}}{g_{\mathrm{LSE}}} \prod_{\imath=1}^{p} \frac{\omega_{\mathrm{LS}}^{(2)}}{\omega_{\mathrm{HS}}^{(\imath)}} \mathrm{e}^{-\frac{\Delta\left(n_{\mathrm{HS}}\right)}{k_{\mathrm{B}} T}}
$$

Equation (18) reduces the problem to that of a set of two-level systems, the degeneracy ratio of which has become:

$$
\left(\frac{g_{\mathrm{HS}}}{g_{\mathrm{LS}}}\right)_{\mathrm{LF}}=\frac{g_{\mathrm{HSE}}}{g_{\mathrm{LSE}}} \prod_{i=1}^{p} \frac{\omega_{\mathrm{LS}}^{(i)}}{\omega_{\mathrm{HS}}^{(i)}}
$$

However, the temperature dependences of the "degeneracies" of the two levels will result in an additional contribution to the entropy, and consequently to the specific heat. The following relation continues to hold:

$$
\Delta S_{\mathrm{LF}}\left(T_{\mathrm{c}}\right)=R\left(\operatorname{Ln} \frac{g_{\mathrm{HSE}}}{g_{\mathrm{LSE}}}+\sum_{\imath=1}^{p} \operatorname{Ln} \frac{\omega_{\mathrm{LS}}^{(2)}}{\omega_{\mathrm{HS}}^{(2)}}\right)=R \operatorname{Ln}\left(\frac{g_{\mathrm{HS}}}{g_{\mathrm{LS}}}\right)_{\mathrm{LF}}
$$

The contributions of low frequencies to $\Delta C_{\mathrm{P}}$, on the contrary, will be weak because, for the harmonic oscillator $C_{\mathrm{P}} \simeq R$ for any $\omega$, in the high temperature limit.

The high frequencies are expected to contribute little to $\Delta C_{\mathrm{P}}$ and $\Delta S\left(T_{\mathrm{C}}\right)$, because the ground vibrational level is little populated when $\hbar \omega \gg k_{\mathrm{B}} T$. 


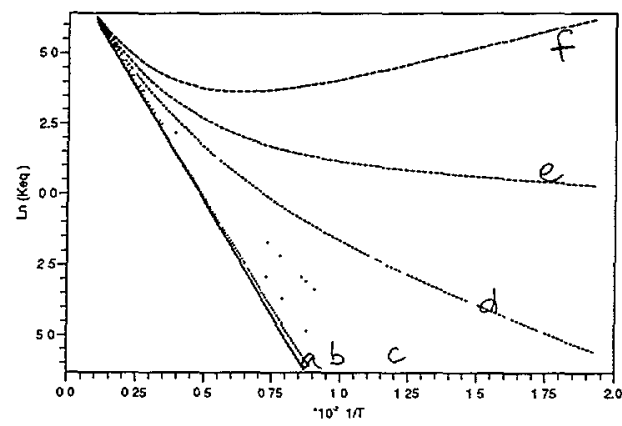

Fig. 3. - Thermal variation of the equilibrium constant, computed in absence of cooperativity, using equation (15) accounting for vibrations: $\hbar \bar{\omega}=100(\mathrm{a}), 200(\mathrm{~b}), 300(\mathrm{c}), 400(\mathrm{~d}), 500(\mathrm{e}), 600$ (f) K. The computed values for situations from (a) to (e) are: $T_{\mathrm{C}}=204.7,194.3,174.9,140.0 \mathrm{~K} ; \Delta S\left(T_{\mathrm{C}}\right)=63.0$, $59.7,53.1,40.0 \mathrm{~J} \mathrm{~K}^{-1} \mathrm{~mol}^{-1} ; \Delta C_{\mathrm{p}}\left(T_{\mathrm{C}}\right)=2.0,8.1,18.7,31.7 \mathrm{~J} \mathrm{~K}^{-1} \mathrm{~mol}^{-1}$. The full line represents the limiting behavior in the low-frequency approxımation, computed with $g_{\mathrm{HS}} / g_{\mathrm{LS}}=2189=5\left(\omega_{\mathrm{LS}} / \omega_{\mathrm{HS}}\right)^{p}$, leading to $\Delta S\left(T_{\mathrm{C}}\right)=\Delta S^{0}=64 \mathrm{~J} \mathrm{~K}^{-1} \mathrm{~mol}^{-1}$

Computations have been performed, in the absence of cooperativity, with the simplifying assumption of an average vibration mode 15 -fold degenerate (15 is the number of distortion modes of the octahedron surrounding the metal atom). We show in Figure 3 the results obtained with the average frequency ratio $\frac{\omega_{L S}}{\omega_{\mathrm{HS}}}=1.5$, leading to (as a first approach) $\Delta S_{\mathrm{LF}}\left(T_{\mathrm{C}}\right)=64 \mathrm{~J} \mathrm{~K}^{-1} \mathrm{~mol}^{-1}$, which is in the middle of the range of the literature data.

For convenience, we defined a quadratic average frequency $\bar{\omega}=\sqrt{\omega_{\mathrm{LS}} \omega_{\mathrm{HS}}}$.

The value given to the electronic degeneracy ratio was the spin-only value, corresponding to the frequent situation of an almost complete quenching of the orbital moment, evidenced for example by the essentially temperature independent and large value of the quadrupole splitting of the HS component of the Mössbauer spectra.

It is worth noting that for cases (e, $f$ ) in Figure 3, the equilibrium temperature is not reached; this results from a high-spin vibronic ground state, obtained when:

$$
\Delta<\frac{p}{2} \hbar\left(\omega_{\mathrm{LS}}-\omega_{\mathrm{HS}}\right)=\Delta_{\text {crit }} .
$$

Figure 3 shows a systematic departure from the linear plot. The latter is only obtained as an asymptotic behavior, for $T \rightarrow 0$ or $\infty$. At intermediate temperatures, such that $\hbar \omega \simeq k_{\mathrm{B}} T$, a positive curvature is observed. Such a distortion is clearly distinguished from a cooperative effect (shown in Fig. 2). It is weak, however, and will be discussed in more details in the next section.

For $\omega \rightarrow 0$, the system behaves as a two-level system with an increased degeneracy ratio (this is the low-frequency approximation).

The values of $\Delta S$ and $\Delta C_{\mathrm{P}}$ (assuming complete conversion for both quantities) were easily obtained by difference between the properties of the $15 \mathcal{N}$ oscillators in the HS or the LS state.

It will be noted that for $r$ close to $1, \Delta C_{P}$ varies almost linearly as a function of $(r-1)$ $\simeq \operatorname{Ln}(r)$ (Fig. 4). The low frequency $(u \ll 1)$ approximation is then:

$$
\Delta C_{\mathrm{P}} \simeq \frac{5}{4} R u^{2} \operatorname{Ln} r
$$

The entropy contribution of the osclllators can be numerically obtained by integrating $\frac{C_{\mathrm{P}}}{T}$. 


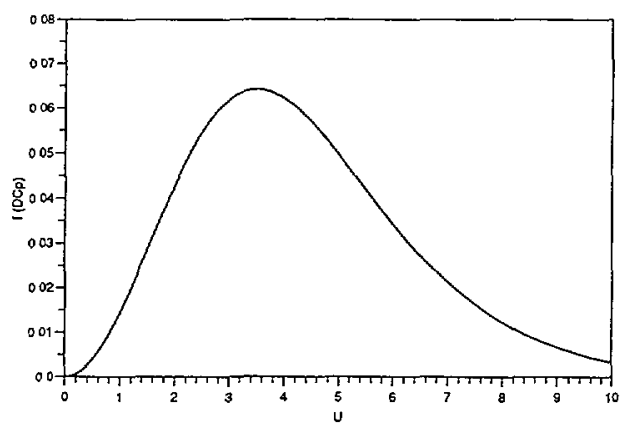

Fig. 4. - Computed variation of $\frac{\Delta C_{\mathrm{P}}}{15 R}$ vs. the reduced variable $u=\frac{\hbar \bar{\omega}}{k_{\mathrm{B}} T}$, for $r=\frac{\omega_{\mathrm{LS}}}{\omega_{\mathrm{HS}}}=1.1$.

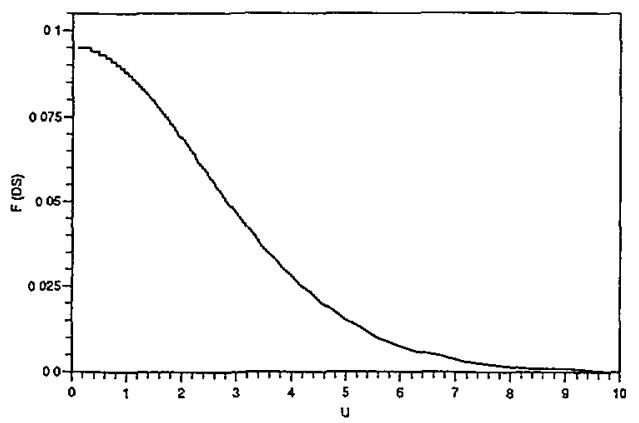

Fig. 5. - Computed variation of $\frac{\Delta S(T)}{15 R}$ vs. the reduced variable $u=\frac{\hbar \bar{\omega}}{k_{\mathrm{B}} T}$, for $r=\frac{\omega_{\mathrm{LS}}}{\omega_{\mathrm{HS}}}=1.1$.

The computed variation of $\Delta S$ is shown in Figure 5. Simlarly to $C_{\mathrm{P}}$ it is almost proportional to $\operatorname{Ln} r$ for $r$ close to 1 . The low-frequency approximation value of $\Delta S$ (Eq. (20)) is, of course, obtained for $\omega \rightarrow 0$.

\section{Discussion}

The presently available experimental vibration spectroscopy data are very incomplete. The only modes correctly identified and measured are the octahedron modes based on the stretching of the Fe-N bonds. In the case of $\mathrm{Fe}(\mathrm{Phen})_{2}(\mathrm{NCS})_{2}[12]$, for example, these frequencies are:

$\omega(\mathrm{Fe}-\mathrm{NCS})=252 \mathrm{~cm}^{-1}$ in the HS state

$=533,528 \mathrm{~cm}^{-1}$ in the LS state

$\omega($ Fe-phen $)=220 \mathrm{~cm}^{-1}$ in the HS state

$=379 \mathrm{~cm}^{-1}$ in the LS state

For the same complex, which undergoes a quasi-complete spin transition at $T_{\mathrm{C}}=176.3 \mathrm{~K}$, the calorimetric data are[5]:

$$
\Delta C_{\mathrm{P}}=18.7 \mathrm{~J} \mathrm{~K}^{-1} \mathrm{~mol}^{-1} ; \quad \Delta S=48.8 \mathrm{~J} \mathrm{~K}^{-1} \mathrm{~mol}^{-1} ;
$$

leading to, in the low-frequency approximation: 


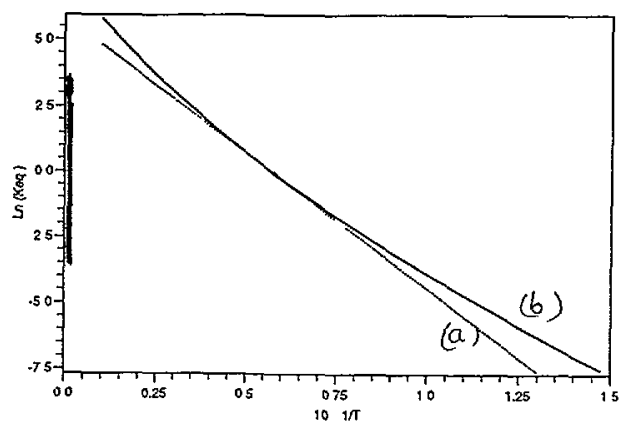

Fig. 6. - Arrhenius plot of the equilibrium constant of a non-interacting( or diluted) system having the same intramolecular data as $\mathrm{Fe}(\mathrm{phen})_{2}(\mathrm{NCS})_{2}$ : a) low-frequency treatment b) complete treatment.

$$
\Delta=\Delta_{0}=\frac{\Delta H^{0}}{\mathcal{N}}=\frac{T_{\mathrm{C}} \Delta S\left(T_{\mathrm{C}}\right)}{\mathcal{N}} \simeq 1034 \mathrm{~K}
$$

Calculations accounting for the six intramolecular modes based on the Fe-N stretching, including $\Delta S_{\text {elect }}=13.4 \mathrm{~J} \mathrm{~K}^{-1} \mathrm{~mol}^{-1}$ yleld:

$$
\Delta S=31.2 \mathrm{~J} \mathrm{~K}^{-1} \mathrm{~mol}^{-1}, \quad \Delta C_{\mathrm{P}}=20.2 \mathrm{~J} \mathrm{~K}^{-1} \mathrm{~mol}^{-1}
$$

An almost correct value is obtained for $\Delta C_{\mathrm{P}}$. The missing contribution to $\Delta S$ can be found by introducing 9 further intramolecular modes of lower frequencies, with the ratio $r=\frac{\omega_{\mathrm{LS}}}{\omega_{\mathrm{HS}}} \simeq 1.26$. Such a values does not seem unrealistic, compared to the ratio $\sim 2$ exhibited by the distortion modes likely to be the most affected by the spin conversion.

We then computed the thermal variation of the equilibrium constant, for an hypothetical similar system when the interactions are switched off. The resulting curve, plotted in Figure 6 , exhibits a weak deviation with respect to the straight line which very likely remains at the limit of the experimental accuracy. The expermmental reachable range is indicated by a vertical bar.

It must be stressed that the accurate determination of the spin populations can present serious difficulties: presence of residual fractions at high or low temperature, slowing down of the kinetics of the spin equilibrium at low temperature[12], dynamic spectra at high temperature. We have observed on several examples that, in case of dynamic spectra, the value given to the Mössbauer linewidth might strongly influence the values obtained for the fitted spin populations.

As a matter of fact, few experimental results, on diluted systems, have provided evidence for departures from the linear Arrhenius plot. We use, as a textbook example, the very accurate data published for Spiering et al. [13] on the diluted complex $\left.\left[\mathrm{Fe}_{0.01}^{\mathrm{II}} \mathrm{Zn}_{0.99} \text { (2-pic) }\right]_{3}\right] \mathrm{Cl}_{2}$. EtOH: Figure 7 compares, in the usual vs. $T$ axes, the best fits using the two-level model and the present model. The improvement is clear. Figure 8 shows a similar comparison, performed using the Arrhenius plot. The vibrational curvature of the data is obvious.

It is worth remarking that the Arrhenius coordinates put an emphasis on the extreme parts of the curve. The resulting zoom-effect reveals a low-temperature misfit of the model, which, however, is likely to remain below the threshold of experimental accuracy, due to the large relative errors associated with the very small experimental values of the HS fraction (0.015 


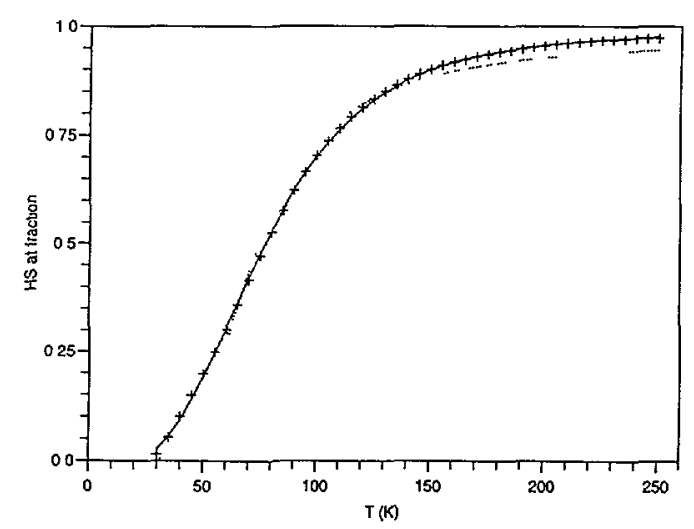

Fig. 7. - Experimental data of $\left[\mathrm{Fe}_{0.01} \mathrm{Zn}_{099}(2-\mathrm{pic})_{3}\right] \mathrm{Cl}_{2}$.EtOH (smoothed values taken in [13]). The best fitted values are: i) two-level model( dotted line): $\Delta_{0}=301 \mathrm{~K}, g_{\mathrm{HS}} / g_{\mathrm{LS}}=56.6 \mathrm{ii}$ ) present model (full line): $\Delta_{0}=977 \mathrm{~K}, \omega_{\mathrm{LS}}=366 \mathrm{~K}, \omega_{\mathrm{LS}} / \omega_{\mathrm{HS}}=1.43$.

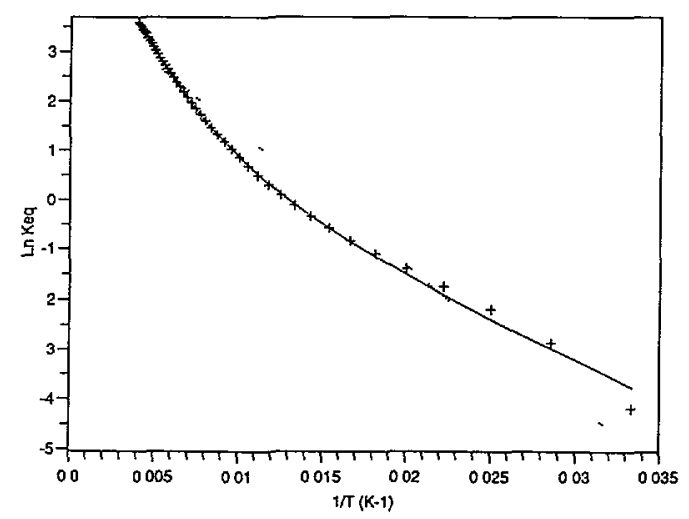

Fig. 8. - Arrhenius plot of the data of Figure 7. The best fitted values are: 1) two-level model (dotted line): $\Delta_{0}=272 \mathrm{~K}, g_{\mathrm{HS}} / g_{\mathrm{LS}}=60.6 \mathrm{ii}$ ) present model (full line): $\Delta_{0}=856 \mathrm{~K}, \omega_{\mathrm{LS}}=332 \mathrm{~K}$, $\omega_{\mathrm{LS}} / \omega_{\mathrm{HS}}=1.39$; further refinements, involving the remaining parameters $J,\left(g_{\mathrm{HS}} / g_{\mathrm{LS}}\right)_{\text {electron. }}$, or small residual HS or LS fractions, did not provide any further significant improvement.

for the lowest temperature). We therefore conclude that the present electrovibrational model successfully fits the data of reference [13].

Also, the (average) vibrational data deduced from the fits are in excellent agreement with expectations:

1) the $\omega_{\mathrm{LS}} / \omega_{\mathrm{HS}}$ ratio (1.43) is smaller than the literature data $(2-2.5)$, determined for the stretching distortion modes,

ii) the $\Delta S$ value, computed at $75 \mathrm{~K}$ (spin equilibrium temperature) $\sim 35 \mathrm{~J} \mathrm{~K}^{-1} \mathrm{~mol}^{-1}$, is consistent with the range of literature values.

The systems where a large vibrational effect may be expected a priori are those having a low equilibrium temperature i.e., a small $\Delta_{0}$, so that the energy scheme is essentially determined by the vibration frequencies. Such a situation is close to the critical situation corresponding to 


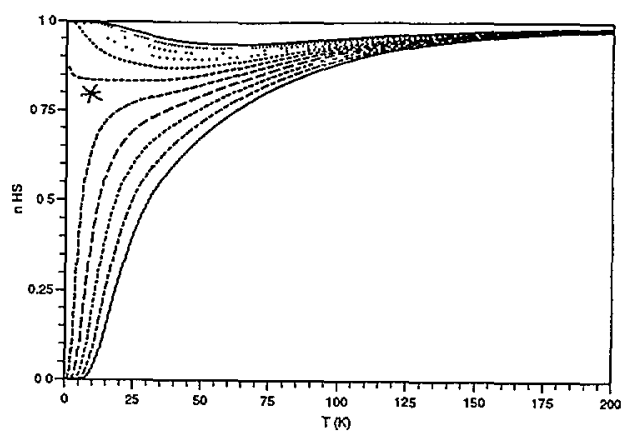

Fig. 9. - Thermal variation of the high-spin fraction, computed close to the critical situation, with the same vibrational data as for Figure 6 , and $\Delta$ ranging from 930 to $1030 \mathrm{~K}$; the critical value is $\simeq 980 \mathrm{~K}$ (curve labeled by a star).

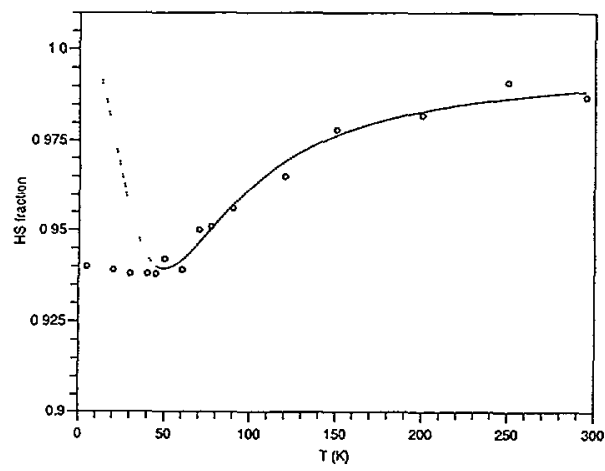

Fig. 10. - Experimental conversion curve of $\mathrm{Fe}^{\mathrm{II}}(\mathrm{TRIM})_{2} \mathrm{~F}_{2}$, where TRIM=4'-(4-methylimidazole) [14], with the best-fit curve using the present electron-vibrational model. The low temperature branch of the computed (equlibrium) curve is not expermentally reached because of the slow kinetics of the spin conversion; it was given a null statistic weight for the fit. The parameter values which fit the equilibrium branch data $(T \geq 50 \mathrm{~K})$ are $\Delta_{0}=356 \mathrm{~K}, \omega_{\mathrm{LS}}=232 \mathrm{~K}, \omega_{\mathrm{LS}} / \omega_{\mathrm{HS}}=1.30$. The electro-vibrational ground state is HS; the first excited level is a low-lying ( $\sim 43 \mathrm{~K})$ LS state.

the change of nature of the vibronic groundstate. We show in Figure 9 the thermal variation of the high-spin fraction, computed for situations close to the critical value (using the complete set of intramolecular vibrations previously estimated for Fig. 6). Similar plots were given by Zimmermann and Konıg [9] using their sophisticated model.

When completing the present study, we discovered a novel experimental case just corresponding to this critical situation, which provides [14] the first example of a spin conversion monitored by the molecular vibrations. The experimental data and their best fit using the present model are shown in Figure 10. A peculiarity arises from the slow kinetics of the spin conversion at low temperature: all data below $\sim 50 \mathrm{~K}$ correspond to the "frozen" system, and therefore depart from the equilibrium computed curve. As expected, the fitted values of the (average) parameters: $\omega_{\mathrm{LS}}=232 \mathrm{~K}, \frac{\omega_{\mathrm{LS}}}{\omega_{\mathrm{HS}}}=1.30$ lead to a reasonable value: $\Delta S(150 \mathrm{~K})=40 \mathrm{~J} \mathrm{~K}^{-1} \mathrm{~mol}^{-1}$

Due to the low temperature "spin freezing", all experimental cases near the critical situation are expected to exhibit an incomplete conversion, with a very large HS fraction at low temperature. For the huge majority of cases, reasonably far from this critical situation, and 
characterized by an almost complete spin conversion, the conclusions of Figures 6 and 7 should hold, so that the low-frequency approxumation can be used. This a posteriori justifies the use of a two-level model with a large degeneracy ratio, as a good first approach. Usual orders of magnitude are derived, following equation (2), from the $\Delta S$ values:

$$
35<\Delta S<80 \mathrm{~J} \mathrm{~K}^{-1} \mathrm{~mol}^{-1} \leftrightarrow 70<\frac{g_{\mathrm{HS}}}{g_{\mathrm{LS}}}<15000
$$

In other words, the two-level model is well suited to describe the electro-vibrational character of the spin conversion, when the latter is (almost) complete. In case of strong cooperativity, an almost complete spin conversion occurs within a narrow temperature range. As far as the intramolecular properties are concerned, the simple two-level electrovibrational model is convenient. Of course, the correct description of the intermolecular interactions remains an open question, for which the Ising model, treated in mean-field approximation, is but a first approach (this may include intramolecular vibrations, following the present model).

A further application of the electron-vibrational character of the two-level model, consists in a purely vibrational coupling model. Cooperativity in two-level systems implies defining the dependence of the parameters of each two-level system on the state of the neighboring system (short range interactions) and on the state of the lattice (long range interactions). This has been done so far by assuming that the energy spacing $\Delta$ depends on the neighboring spin state (Eq. (8)). Alternatively, it may be assumed that the vibration frequencies are sizably affected, and accordingly we consider variable degeneracies depending on the neighboring spin state. In a mean-field approach, the ratio is taken as:

$$
\frac{g_{\mathrm{HS}}}{g_{\mathrm{LS}}}=\left(\frac{g_{\mathrm{HS}}}{g_{\mathrm{LS}}}\right)_{\mathrm{eq}} \mathrm{e}^{\eta\left(n_{\mathrm{HS}}-\frac{1}{2}\right)}
$$

Equation (23) is written in such a way as to give the vibrational coupling parameter $\eta$ the following meaning

$$
\eta=\sum_{\imath=1}^{15} \operatorname{Ln} \frac{\left(\frac{\omega_{\mathrm{LS}}^{(2)}}{\omega_{\mathrm{HS}}^{(2)}}\right) \mathrm{HS}}{\left(\frac{\omega_{\mathrm{LS}}^{(2)}}{\omega_{\mathrm{HS}}^{(2)}}\right) \mathrm{LS}}
$$

where the outer indices HS, LS stand for the state of the lattice surrounding the considered molecule, and the inner indices LS, HS, as usual are the spin state of the molecule itself. A positive value of $\eta$ is associated with a cooperative effect. The equilibrium constant is then obtained as:

$$
K_{\text {eq }}(T)=\left(\frac{g_{\mathrm{HS}}}{g_{\mathrm{LS}}}\right)_{\mathrm{eq}} \mathrm{e}^{-\frac{\Delta_{0}}{k_{\mathrm{B}} T}+\eta\left(n_{\mathrm{HS}}-\frac{1}{2}\right)}
$$

Equation (25) gives the coupling term an entropic form (as in Ref. [9]). This obviously comes from the "vibrational" degeneracies (or densities of states) which directly contribute to entropy.

Such an entropic coupling model yields conversion curves and first-order transitions as does the usual enthalpic model. The threshold value for the coupling parameter is $\eta_{\text {thres }}=4$. It is clear that in the case of a strong cooperativity, the temperature range where the conversion occurs is narrow, and the difference between enthalpic and entropic forms become minor. No detailed comparıson is given here of the enthalpic and entropic forms of cooperativity; a 
relevant discussion would prerequisite a detailed knowledge of the vibrational properties upon conversion, and the ability to distinguish between internal and external origins of the changes in the molecular vibrations.

\section{Conclusion}

The effect of intramolecular vibrations, on the spin conversion of independent molecules, has been studied in the frame of the two-level model, completed by a set of harmonic oscillators. This provides an explanation of the high value of the molar entropy increase $\Delta S$ upon LS $\rightarrow$ HS conversion.

In most cases, the thermal variation of the spin population can be calculated using the simple two-level model with high degeneracy values implicitly accounting for intramolecular vibrational effects. In such a case, only global information can be derived from the data:

i) from $\Delta S$ (or equivalently from the high-temperature conversion curve), an expression involving both the number of modes and the average frequency ratio $\frac{\omega_{L S}}{\omega_{\mathrm{HS}}}$,

ii) from $\Delta C_{\mathrm{P}}$, an additional information which also involves the values of the frequencies.

We have shown a novel example, for which the lowest HS and LS electro-vibrational states are almost equi-energetic. Then only considering as an average the 15 octahedron distortion modes, we could reproduce the conversion curve with reasonable values of the vibrational parameters. The latter monitor the shape of the conversion curve.

We also have re-interpreted the accurate data of Spiering et al. on spin conversion in $\left[\mathrm{Fe}_{0.01} \mathrm{Zn}_{0.99}(2-\mathrm{pic})_{3}\right] \mathrm{Cl}_{2} . \mathrm{EtOH}$, and given evidence for a typical vibrational effect in this system, with the help of an Arrhenius plot.

\section{Acknowledgments}

We are indebted to J. Zarembowitch, M. Verelst, K. Boukheddaden and J. Linares for useful discussions and valuable help. This work was supported by the "Molecular Magnetism" European Community Network.

\section{References}

[1] Slichter C.P. and Drickamer H.G., J. Chem. Phys. 56 (1972) 2142.

[2] Wajnflasz J. and Pick R., J. Phys. IV France 32 (1971) C1-91.

[3] Barı R. and Sivardière J., Phys. Rev. B5 (1972) 4466.

[4] Bousseksou A., Nasser J., Linares J., Boukheddaden K. and Varret F., Mol. Cryst. Liq. Cryst. 234 (1993) 269.

[5] Soral M. and Seki S., J. Phys. Chem. Solids 35 (1974) 555.

[6] Claude R., Real J.A., Zarembowitch J., Kahn O., Ouahab L., Grandjean D., Boukheddaden K., Varret F. and Dworkin A., Inorg. Chem. 29 (1990) 4442.

[7] Toftlund H., Coord. Chem. Rev. 94 (1989) 67.

[8] Könı E., Struct. Bond. 76 (1991) 51.

[9] Zimmermann R. and Könıg E., J. Phys. Chem. Solıds 38 (1977) 779. 
[10] Kambara T., J. Chem. Phys. 70 (1979) 4199.

[11] Bousseksou A., Nasser J., Linares J., Boukheddaden K. and Varret F., J. Phys. I France 2 (1992) 1381;

Bousseksou A., Varret F. and Nasser J., J. Phys. I France 3 (1993) 1463;

Lemercier G., Bousseksou A., Seigneuric S., Varret F. and Tuchagues J.P., Chem. Phys. Lett. 226 (1994) 289.

[12] Takemoto J.H. and Hutchinson B., Inorg. Nucl. Chem. Lett. 8 (1972) 769;

Hauser A., Gütlich P. and Spiering H., Inorg. Chem. 25 (1986) 4245;

Decurtins S., Gütlich P., Kasselbach K.M., Hauser A. and Spierıng H., Inorg. Chem. 24 (1985) 2174;

Gütlich P., Köpen K. and Steinhauser H.G., Chem. Phys. Lett. 74 (1980) 475.

[13] Jacobi R., Spierıng H. and Gütlich P., J. Phys. Chem. Solids 53 (1992) 267.

[14] Bousseksou A., Verelst M., Constant-Machado H., Lemercier G., Tuchagues J.P. and Varret F., submitted to J. Am. Chem. Soc. 